\title{
Designing Shift Handoff Software: Clinical Learners and Design Students Collaborate Using the "Design Thinking" Process
}

\author{
Blake LESSELROTH ${ }^{\mathrm{a}, 1}$, Hannah PARK ${ }^{\mathrm{b}}$, Helen MONKMAN ${ }^{\mathrm{c}}$ Ashten DUNCAN $^{\mathrm{a}}$, \\ Gabriel THOMPSON ${ }^{\mathrm{a}}$, and Ryan YARNALL ${ }^{\mathrm{a}}$ \\ a'University of Oklahoma, School of Community Medicine, Tulsa, Oklahoma, USA \\ ${ }^{b}$ School of Architecture and Design, University of Kansas, Lawrence, Kansas, USA \\ ${ }^{\mathrm{c}}$ School of Health Information Science, University of Victoria, Victoria, Canada
}

\begin{abstract}
Handoffs in patient care responsibilities between practitioners are common in the hospital setting. Because inadequate communication can lead to patient harm, professional organizations have published recommendations and practical guides to support standardized workflow. However, currently available electronic medical record (EMR) tools rarely provide the requisite functionality to support work and often suffer from major usability flaws. Our internal medicine residency program sponsored a quality improvement initiative to improve the design of handoff tools. To support this initiative, our medical informatics program collaborated with a school of architecture and design to identify requirements and ideate interface prototypes. In this article, we describe how we used Design Thinking principles and methods to inform our product design lifecycle, create novel designs, and teach inter-professional students health systems science concepts.
\end{abstract}

Keywords. Human-Computer Interface, Ergonomics, Patient Handoff, Electronic Health Records, User-Centered Design, Design Thinkin

\section{Introduction and Problem Statement}

When healthcare professionals reach the end of a shift, it is customary to communicate patient care responsibilities to their colleagues [1]. These handoffs are commonplace in the hospital setting and generally follow a standardized protocol wherein the outgoing party provides critical status updates, pertinent background information, and tasks or plans to the incoming party [2]. Clinical evidence suggests that high-quality communication during handoffs can improve care, whereas inadequate communication can lead to errors and adverse events [3]. Unfortunately, various human and system-level factors can hinder communication, including communicator fatigue, competing priorities, environmental distractions, and subpar health information support systems [4].

Resident physicians across all specialties hand off patient lists daily. For this reason, graduate medical training accreditation standards in the United States require programs to instruct trainees in standardized handoff methods, provide tools to support handoff

\footnotetext{
${ }^{1}$ Lesselroth, Blake, Department of Health Informatics, School of Community Medicine, University of Oklahoma-University of Tulsa, 4502 E. $41^{\text {st }}$ Street, Tulsa, Oklahoma, 74135, USA; E-mail: BlakeLesselroth@ouhsc.edu.
} 
activities, and evaluate the handoff quality [5]. While there is no universally accepted standard for conducting shift handoffs, the most common method for training residents involves structured, bidirectional oral communication with a "read-back" step. Handoff aids and guidelines have been widely published, and many electronic medical records (EMRs) include embedded tools to support a standardized workflow [6]. Despite these efforts, residents do not always follow guidelines, and most EMR tools suffer from usability issues [7]. There is an urgent need to leverage and improve EMR handoff tools to increase the success of these critical moments in the continuity of patient care.

There is neither robust evidence to inform the design of handoff tools, nor clear guidance for implementation within EMR interfaces. Therefore, there is a need for more rigorous user-experience (UX) research and evidence-based design. We describe a collaboration between our medical and design schools and the first steps in a multi-phase process to assemble design requirements and generate early interface prototypes. The purpose of this paper is to (1) review the Design Thinking (DT) framework relative to medical and health informatics development [8]; (2) describe how we engaged learners on an interprofessional project to design improved handoff technology; and (3) share our evaluative methods and early findings.

\section{Theory}

DT is a framework for developing human-centered products, services, and strategies that emerged from the fields of design and user experience (UX) [9]. DT encompasses a highlevel process flow that encourages iterative exploration of candidate solutions, rapid prototyping, and rigorous product testing. It approaches the practice of creating products and customer experiences as a science, providing an array of practical techniques for planning, defining, and delivering innovations. While there several published versions of DT, we adapted one developed at Stanford and the Hasso-Plattner Institute [10]. This version has of five steps: (1) empathize; (2) define; (3) ideate; (4) prototype; and (5) test.

\section{Methods}

\section{Empathize: Conducting a Rapid Ethnography}

The goal of this phase is to empathize with users and understand their tasks, goals, and pain points [9]. Our research team, which included medical students and residents, conducted an abbreviated ethnography using Ash's rapid assessment process (RAP) [11]. RAP entails analysis of field notes gathered using mixed-methods, including direct observations, subject interviews, workflow mapping, and artifact analysis. It encourages including representative end-users on the research team to provide needed context.

\section{Define: Drafting Requirements and Needs Statements}

The define phase articulates usability problems to formulate design [9]. We organized requirements into a set of user needs statements using recommendations from the medical literature, handoff guidelines, and our ethnography findings. Needs statements written during the "define" phase included a target user description, the user's need, and why the 
need is important [12]. The needs statement syntax forces developers to summarize the problem, consider a metric for success, and gain deeper insight into users' goals.

\section{Ideate: Creating a wide range of early user interface concepts}

In this phase, the goal is to generate many problem solutions [9]. Internal medicine faculty provided to an undergraduate design class a client brief that included background information about handoffs, the context of use in our residency program, the problem statement, data requirements, and our user needs statements. Using videoconferencing, faculty met with students to provide an overview of the project, demonstrate current tools, and answer questions. Design faculty instructed students to create four deliverables: (1) interface designs illustrating desktop and smartphone configurations; (2) a presentation board describing the software functionality and site map; (3) a video demonstrating software use; and (4) a practice pitch explaining to stakeholders the business case, problem statement, and software solution. The faculty used a quantitative usability scoring rubric adapted from the System Usability Scale to score six dimensions (i.e., efficiency, effectiveness, safety, aesthetics, usefulness, and ease-of-use) for each design during the pitch [13]. We averaged the scores across dimensions to calculate an overall score. Evaluators could assign a maximum score of 20.

\section{Results}

\section{Define: Design requirements}

Our design brief for students included general specifications and a needs statement table. The specifications included two user personas, a description of the technology ecosystem, a data dictionary including 16 required data elements, and usability benchmarks. The table included 18 unique needs statements. (Table 1).

Table 1. An excerpt from the design brief showing requirements arranged in a "needs-statement" format.

\begin{tabular}{|c|c|c|c|c|}
\hline A user & $\begin{array}{c}\text { needs a } \\
\text { way to }\end{array}$ & do something & so that & $\begin{array}{c}\text { a need is satisfied or a goal is } \\
\text { met }\end{array}$ \\
\hline A medicine resident & $\begin{array}{l}\text { needs a } \\
\text { way to }\end{array}$ & $\begin{array}{l}\text { to import patient data } \\
\text { from the EMR }\end{array}$ & so that & $\begin{array}{l}\text { he/she never makes a } \\
\text { transcription error when } \\
\text { updating the list }\end{array}$ \\
\hline A medicine resident & $\begin{array}{l}\text { needs a } \\
\text { way to }\end{array}$ & $\begin{array}{l}\text { to communicate } \\
\text { required data elements } \\
\text { to a colleague }\end{array}$ & so that & $\begin{array}{l}\text { a health issue is quickly and } \\
\text { correctly managed when cross- } \\
\text { covering patients }\end{array}$ \\
\hline A medicine resident & $\begin{array}{l}\text { needs a } \\
\text { way to }\end{array}$ & $\begin{array}{l}\text { see all patients in a } \\
\text { single view }\end{array}$ & so that & $\begin{array}{l}\text { he/she can scan for a specific } \\
\text { patient or action item quickly } \\
\text { when on the hospital wards }\end{array}$ \\
\hline
\end{tabular}

\section{Ideate: Sample user interface designs}

The goal of this phase is to iteratively develop prototypes over a series of work sprints [9]. The students developed 34 presentation boards and 68 unique interface designs simulating desktop and smartphone experiences. We show a sample student deliverable in Figure 1. The average total usability score for the students' designs was 15.2; the mode was 15.6 (minimum score: 13.3; maximum score 19.4). 

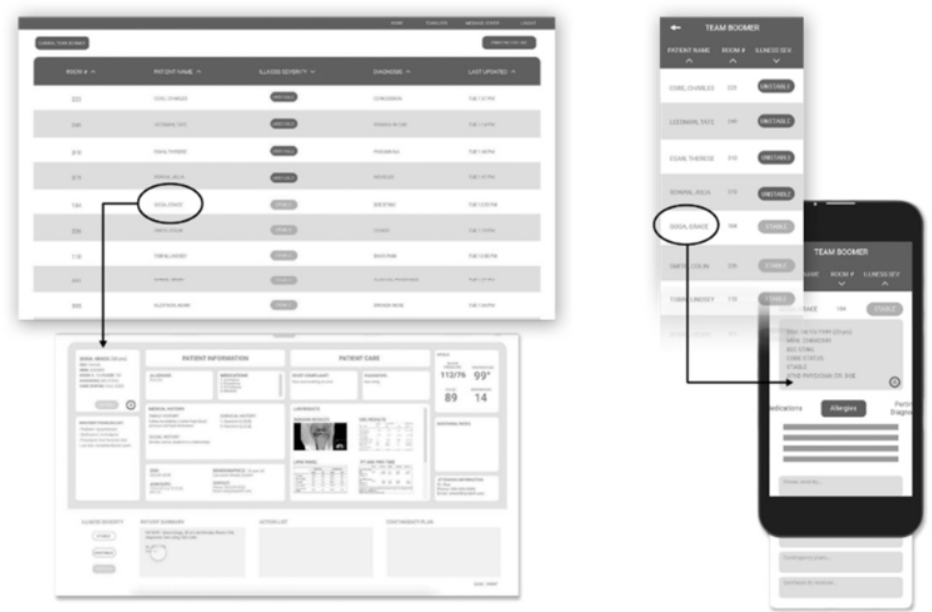

Figure 1. Examples of desktop and smartphone interface concepts for the shift handoff tool. ${ }^{2}$

\section{Discussion, Next Steps and Conclusions}

In this monograph, we describe how we used a DT process and leveraged student talents from several disciplines to generate a large number of shift handoff tool prototypes. All the prototypes included the data elements required by handoff best-practice recommendations and many offered a more intuitive and workflow-compatible UX as compared to current tools. Our preliminary scoring suggests that several designs would be suitable for prototyping. However, we still need to validate and test these designs. In our next phase, we will develop a working prototype to simulation test with clinicians using representative use-cases. We anticipate measuring products against published usability benchmarks and current-state systems.

This work illustrates how to apply DT to health record development, medical education, and interprofessional collaboration. Despite the promise EMRs hold to improve efficiency and performance, they often fail to meet user expectations and are seen as a major contributor to clinician burnout [14]. Rather than waiting for EMR vendors to optimize UX, stakeholders can be agents of change. Herein, we described ways to operationalize DT throughout the product lifecycle. Our methods are relatively low cost and can help democratize the development process, enabling informaticians, clinicians, and other stakeholders to play key roles in quality improvement initiatives.

This project also demonstrates a novel "learning lab" for the instruction of clinical informatics, person-centered design, and health systems science. The modern health delivery setting is a complex, adaptive system; clinicians need to know more than medical science to navigate the social, environmental, and technical dimensions of care [15]. To improve care quality and the patient experience, future health professionals will need to analyze systems, develop scalable solutions, and lead meaningful change. To promote this broader view of professionalism, accreditation organizations in the United

\footnotetext{
${ }^{2}$ Designs by Grace Goga, School of Architecture and Design, University of Kansas
} 
States have published educational program standards that emphasize systems-based practice, interprofessional collaboration, health technology competency, and continuous quality improvement methods [15]. This collaborative educational practicum between two universities gave learners hands-on experience developing competencies while solving a real patient safety issue. Students engaged in participatory learning by reviewing quality improvement and clinical informatics literature, completing a gap analysis, collecting and analyzing ethnographic data, drafting technical requirements, and designing technology concepts.

We hope this work provides a blueprint for similar interprofessional collaborations. This design assignment represents the beginning of an ongoing relationship between medical and design schools at two different academic centers. Solutions to global healthcare challenges demand transdisciplinary teams and innovative design. Population health is governed as much by social determinants of health and health systems as it is by individual behavior and the natural history of disease. Therefore, as leaders and educators, we must cultivate the habits of systems thinkers, leverage the expertise of other disciplines, and practice the skills required to be effective in teams. This manuscript illustrates a viable and replicable strategy to provide this instruction.

\section{References}

[1] Arora VM, Manjarrez E, Dressler DD, Basaviah P, Halasyamani L, Kripalani S. Hospitalist handoffs: a systematic review and task force recommendations. Journal of hospital medicine: an official publication of the Society of Hospital Medicine. 2009;4(7):433-40.

[2] Lee S-H, Phan PH, Dorman T, Weaver SJ, Pronovost PJ. Handoffs, safety culture, and practices: evidence from the hospital survey on patient safety culture. BMC health services research. 2016;16(1):1-8.

[3] The Joint Commission. Inadequate hand-off communication. Sentinel Event Alert. 2017;58:1-6.

[4] Abraham J, Nguyen V, Almoosa KF, Patel B, Patel VL, editors. Falling through the cracks: information breakdowns in critical care handoff communication. AMIA Annual Symposium Proceedings; 2011: American Medical Informatics Association.

[5] Riesenberg LA, Leitzsch J, Massucci JL, Jaeger J, Rosenfeld JC, Patow C, et al. Residents' and attending physicians' handoffs: a systematic review of the literature. Academic Medicine. 2009;84(12):1775-87.

[6] Davis J, Riesenberg LA, Mardis M, Donnelly J, Benningfield B, Youngstrom M, et al. Evaluating outcomes of electronic tools supporting physician shift-to-shift handoffs: a systematic review. Journal of graduate medical education. 2015;7(2):174-80.

[7] Riesenberg L. Shift-to-Shift Handoff Research: Where Do We Go From Here? Journal of Graduate Medical Education. 2012;4(1):4-8.

[8] Woods L, Cummings E, Duff J, Walker K. Design thinking for mHealth application co-design to support heart failure self-management. Studies in health technology and informatics. 2017;241:97-102.

[9] Dam R, Siang T. What is design thinking and why is it so popular. Retrieved July. 2018;10.

[10] Plattner H, Meinel C, Weinberg U. Design-thinking: Springer; 2009.

[11] Ash JS, Sittig DF, McMullen CK, Guappone K, Dykstra R, Carpenter J, editors. A rapid assessment process for clinical informatics interventions. AMIA Annual Symposium Proceedings; 2008: American Medical Informatics Association.

[12] Gibbons S. User need statements: The 'define' stage in Design Thinking 2019. Available at: https://www.nngroup.com/articles/user-need-statements/. Accessed January 11, 2021.

[13] Sauro J. A Practical Guide to the System Usability Scale: Background, Benchmarks \& Best Practices: CreateSpace Independent Publishing Platform; 2011.

[14] Downing NL, Bates DW, Longhurst CA. Physician burnout in the electronic health record era: are we ignoring the real cause? : American College of Physicians; 2018.

[15] Skochelak SE. Health systems science e-book: Elsevier; 2020. 